\title{
Modeling urban floods at sub-meter resolution: challenges or opportunities for flood risk management?
}

\author{
Gustavo A. M. de Almeida ${ }^{\mathrm{a}, *}$, Paul Bates ${ }^{\mathrm{b},}$, Hasan Ozdemir ${ }^{\mathrm{c}}$ \\ ${ }^{a}$ University of Southampton, Faculty of Engineering and the Environment, SO171BJ, \\ $U K$. \\ ${ }^{b}$ University of Bristol, School of Geographical Sciences, University Rd., Bristol BS8 1SS, \\ $U K$. \\ ${ }^{c}$ Geography Department, Istanbul University, 34459 Istanbul, Turkey.
}

\begin{abstract}
In this article we investigate the influence of fine scale changes in the elevation of urban terrains on the dynamics and final distribution of flood inundation generated by intense rainfall. Numerical experiments have been performed combining $2 \mathrm{D}$ shallow-water model with extremely fine resolution $(10 \mathrm{~cm})$ terrain data. Our results reveal that localized, decimetric-scale alterations in the elevation of streets can lead to remarkable differences in the flood inundation. These results confirm the important role played by finely resolved and accurate terrain data in capturing flow patterns that have a central impact on model predictions of flood inundation. Also, we argue that the observed sensitivity of flood inundation to small-scale topographical features paves the way to new opportunities for flood risk management measures. In particular, engineering flood resilient urban surfaces using fine resolution models has a potential to considerably reduce flood impacts at a relatively low cost.

Keywords: urban flood, modelling, terrestrial LiDAR
\end{abstract}

\section{Introduction}

It is an unfortunate and often tragic combination of factors that places urban flooding amongst the most damaging and costly of all natural hazards. Worldwide, a relatively frequent occurrence of heavy rainfall storms combine with high levels of human exposure and high-value and vulnerable assets to produce multi-billion losses every year. In a world of rapid urbanization and

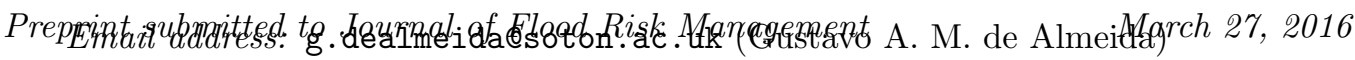


considering the prospect of strongly adverse climate change effects, understanding and mitigating urban flood risks is eliciting widespread concern and has become an issue of the highest priority.

Among different sources of flooding that can occur in urban areas (e.g. river, coastal, groundwater), surface water flooding (i.e. flood resulting from intense excess rainfall) is often responsible for a significant proportion of flood losses. For instance, the Environment Agency of England and Wales estimates that 3.8 million properties are at risk of surface flooding $(E A, 2009)$ in England and Wales. A drastic example of this exposure occurred during the summer of 2007, when approximately two thirds of the 55,000 damaged properties were flooded by surface water (DEFRA, 2008; Evans et al, 2008). In spite of the relevance to current and future generations, a comprehensive understanding of the dynamics of surface water urban inundation, as well as the development of methods to accurately model and mitigate its consequences are still in their infancy when compared to the substantial progress achieved over decades of research in river and coastal flooding. While models of sewerage systems date back to the early 70's (Delleur, 2003), the development and application of the first coupled sewer-surface flow models only emerged during the first decade of the 21st century (Djordjevic et al, 1999). In addition, prevention and mitigation of urban flooding has historically been limited in scope, and almost exclusively linked to the appropriate design and sizing of the sewerage system, a vision that has only recently been broadened to include the concepts of Sustainable Drainage Systems (SuDS). Little attention has been given to a thorough understanding of the role played by urban topography (in particular sub-meter scale) on the behavior of floods. This is despite the fact that under medium to extreme rainfall events (when the sewer system is usually surcharged) most of the flood water is expected to be carried as overland flow (e.g. Mark et al, 2004; Mignot et al, 2006), in which case the layout of surface pathways will largely dictate what areas of the urban terrain will be inundated.

Even though during intense rainfall events large parts of urban areas may be exposed to relatively high flow depths, this usually occurs as a result of the accumulation (in terrain depressions or lowland areas) of water previously routed from the urban catchment along roads and other flow paths. The transport of surface flow along these pathways is a phenomenon of shallow water (i.e. typically $<20 \mathrm{~cm}$ deep) that can move at relatively high velocities. This type of flow is controlled by small-scale features of the urban terrain such as the height of curbs, the shape and dimensions of road cambers, as well as by 
the connectivity of roads and pathways. The road network can be particularly efficient in transporting water across the urban domain and therefore plays an important role in the ultimate distribution of flooded areas. Capturing the effects of these elements in a two dimensional (2D) model requires very fine resolution topography (i.e. sub-meter resolution, as discussed in Ozdemir et al, 2013), which translates into extremely high computational times that are often unfeasible in most practical applications. This results from the fact that the computational time of explicit two-dimensional models usually used for flood simulations scales with the resolution of the mesh raised to the power of three. For instance, refining a mesh from $1 \mathrm{~m}$ to $10 \mathrm{~cm}$ translates into a $1000 \times$ increase in the simulation time.

As a response to the above computational barrier, a number of practical modeling abstractions and simplifications have emerged, which attempt to overcome this limitation and to achieve simulation run times that are compatible with available computational resources. Particular efforts have been devoted to models that conceptualize the surface component of urban floods as a set of elements such as small catchments and/or ponds that are interconnected by $1 \mathrm{D}$ channels that represent the road network (e.g. Mark et al, 2004; Nasello and Tucciarelli, 2005; Maksimovic et al, 2009; Leandro et al., 2009), in a similar way to the first river network models of the late 1970's (e.g. Cunge, 1980). The coupling of this representation of the surface flow with a sewerage network model is often described as a 1D-1D model, as opposed to the 2D-1D approach, in which a two dimensional model is used to simulate the overland component of the flow. Some of the limitations of the 1D representation of surface flow (such as the dependency on user-defined schemes, such as 1D network of pathways and storage elements) have been previously exposed (Mark et al, 2004; Leandro et al., 2009), while other aspects related to the upscaling of sub-meter features remain largely unknown.

Two-dimensional models used in urban flooding are usually based on the shallow water equations (Mignot et al, 2006; Bazin et al, 2014), and simplified forms of these equations such as the zero inertial (e.g. Nasello and Tucciarelli, 2005; Leandro et al., 2009) and local inertial approximations (e.g. Aronica and Lanza, 2005; Fang and Su, 2005; Bates et al, 2010; de Almeida et al, 2012; de Almeida and Bates, 2013), or even simpler formulations (Sampson et al, 2012), have also been widely adopted to speed up simulations. Another strategy to reduce the computational burden of $2 \mathrm{D}$ models focuses on defining sub-grid abstractions that resolve some of the complexities of the urban relief, which is modeled at coarse resolution (e.g. $10 \sim 100 \mathrm{~m}$ ). 
Among this type of models, those adopting the concept of porosity to describe urban features such as buildings have attracted significant attention (e.g. Molinaro et al, 1994; Sanders et al, 2008; Soares-Frazao et al, 2008; Guinot, 2012 to cite but a few). While this approach correctly represents some of the physics operating at intermediate resolution scales (such as the influence of buildings on mass and momentum conservation, which is governed by building dimensions and spacings) and perform well in representing catastrophic flood events (e.g. dam-break induced), it lacks the ability to capture wetting and drying, blockage and other directional effects that are governed by considerably fine scale topographical features.

To date, two dimensional modeling of urban floods has been performed almost exclusively using digital elevation models (DEMs) with resolutions of $1 \mathrm{~m}$ or coarser (e.g. Mark et al, 2004; Fang and Su, 2005; Aronica and Lanza, 2005; Gallegos et al, 2009; Leandro et al., 2009; Maksimovic et al, 2009; Gallien et al, 2011; de Almeida et al, 2012). Advances in computational resources and methods combined with the recent availability of sub-meter resolution terrestrial LiDAR data have enabled the first two-dimensional simulations of urban inundation to be performed at resolutions as low as $10 \mathrm{~cm}$ (Ozdemir et al, 2013). These extremely fine resolution simulations have shown that differences in model predictions persist even as the mesh resolution is refined from $50 \mathrm{~cm}$ to $10 \mathrm{~cm}$. Implicit to this dependency of simulation results on mesh resolution are two different albeit interrelated issues. Firstly, the shape of different terrain features are degraded as the resolution is coarsened, which particularly affects the flow conveyance of road cambers and the storage capacity of different elements (e.g. depression storage). Secondly, and arguably more importantly for shallow water flows, is the fact that the elevation of local peaks are closely approximated at fine resolution, but are in general underestimated at coarser resolution as a result of the increased average distance from the peaks to sampled points. For example, considering a road camber with average cross slope of $4 \%$, the maximum error introduced to the vertical position of the crown by a $5 \mathrm{~m}$ resolution sampling is $10 \mathrm{~cm}$. This is of the same order of magnitude as typical flood depths that are observed at road networks, and is expected to allow the model to incorrectly route water along directions that would be topographically blocked in reality.

If the sensitivity of flood inundation to decimetric-scale elevation changes confirmed, it has two important impacts on the future of flood risk assessment and management. Firstly, it highlights the need for finely resolved and accurate topography, which poses significant challenges to current generation 
computational resources. Secondly, it paves the way for a range of new opportunities for flood risk mitigation that have not been previously explored, and which have the potential to considerably reduce the impacts of extreme storms at relatively low cost.

The value of finely resolved topography in flood inundation modeling is an issue of intense recent debate, particularly when analyzed in the broader context of other sources of uncertainties that are inherently present in practical flood risk assessments (e.g. Dottori et al, 2013 and references therein). While results from grid refinement sensitivity analysis (e.g. Ozdemir et al, 2013) indicate that horizontal resolution plays an important role on model results, it is unclear the extent to which small perturbations in the elevation can produce significant changes to the patterns of surface flood inundation. In this article an extremely fine resolution $(10 \mathrm{~cm})$ description of the urban terrain is combined with a highly accurate and robust finite volume shallow water model to analyze the effects of decimetric scale and localised changes in the topography on the dynamics and outcomes of urban flooding. This relation is explored by introducing small modifications in the elevation of the original $10 \mathrm{~cm}$ resolution DEM, and comparing the simulation results against those obtained with the undisturbed DEM. Even though direct modelling of floods at such fine resolution (i.e. $10 \mathrm{~cm}$ ) is unfeasible for any practical purposes in the foreseeable future, they offer a unique opportunity to clarify the extent to which decimetric scale terrain features control flood dynamics. The results of this analysis are then used to open a discussion on the challenges and opportunities that are intrinsically associated with the topography-impact nexus.

\section{Numerical model}

The model used here is based on the two-dimensional shallow water equations

$$
\frac{\partial \mathbf{U}}{\partial t}+\frac{\partial \mathbf{F}(\mathbf{U})}{\partial x}+\frac{\partial \mathbf{G}(\mathbf{U})}{\partial y}=\mathbf{S}_{1}(x, y, \mathbf{U})-\mathbf{S}_{2}(x, y, \mathbf{U})
$$

where the $\mathbf{U}(x, y, t)$ is the vector of conserved variables, $\mathbf{F}(\mathbf{U})$ and $\mathbf{G}(\mathbf{U})$ are the flux vectors in the $x$ and $y$ directions, respectively, and $\mathbf{S}_{1}(x, y, \mathbf{U})$ and 
$\mathbf{S}_{2}(x, y, \mathbf{U})$ are the slope and friction source terms, respectively:

$$
\begin{gathered}
\mathbf{U}=\left[\begin{array}{c}
h \\
h u \\
h v
\end{array}\right], \mathbf{F}=\left[\begin{array}{c}
h u \\
h u^{2}+\frac{1}{2} g h^{2} \\
h u v
\end{array}\right], \mathbf{G}=\left[\begin{array}{c}
h v \\
h u v \\
h v^{2}+\frac{1}{2} g h^{2}
\end{array}\right], \\
\mathbf{S}_{1}=\left[\begin{array}{c}
0 \\
g h S_{o x} \\
g h S_{o y}
\end{array}\right], \mathbf{S}_{2}=\left[\begin{array}{c}
0 \\
g h S_{f x} \\
g h S_{f y}
\end{array}\right],
\end{gathered}
$$

$h$ is the water depth, $u$ and $v$ are the $x$ and $y$ components of the velocity, $g$ is the acceleration due to gravity, $S_{o x}$ and $S_{o y}$ are the $x$ and $y$ components of the bed slope (i.e. $-\partial z / \partial x$ and $-\partial z / \partial y$, respectively, where $z$ is the bed elevation) and $S_{f x}$ and $S_{f y}$ the corresponding components of the friction slope. The numerical model solves the integral form of eqs. (1):

$$
\frac{\partial}{\partial t} \int_{\Omega} \mathbf{U} d \Omega+\oint_{\partial \Omega}(\mathbf{E} \cdot \mathbf{n}) d l=\int_{\Omega}\left(\mathbf{S}_{1}-\mathbf{S}_{2}\right) d \Omega
$$

where $\mathbf{E}$ is the $3 \times 2$ flux tensor $\mathbf{E}=(\mathbf{F}, \mathbf{G}), \Omega$ and $\partial \Omega$ respectively denote an arbitrary domain and its boundary, and $\mathbf{n}$ is a unit outward vector normal to $\partial \Omega$. Eqs. 2 can be obtained by integrating (1) over $\Omega$ and then applying Gauss's theorem to the integral of the flux terms.

The computational domain is discretised using an unstructured mesh composed of triangular cells (Figure 1). Eqs. 2 are integrated numerically using a first order Godunov finite volume scheme, and a fractional step (e.g. described in LeVeque, 2002). First the cell-averaged value of the conserved variables $\mathbf{U}_{i}$ in cell $\Omega_{i}$ are updated considering the flux terms (homogeneous part) and the bed slope, but neglecting the friction source term. $\mathbf{S}_{1}$ is evaluated with the method of Valiani and Begnudelli (2006), by which the area integral of $\mathbf{S}_{1}$ in (2) is transformed into a boundary integral that can be computed numerically at the edges of the cells. This first step is written as:

$$
\mathbf{U}_{i}^{*}=\mathbf{U}_{i}^{n}-\frac{\Delta t}{A_{i}}\left(\sum_{k=1}^{3}\left(\mathbf{E}^{*}-\mathbf{H}\right)_{i, k}^{n} \mathbf{n}_{i, k} l_{k}\right) \quad ; \quad \mathbf{H}=\left[\begin{array}{cc}
0 & 0 \\
\left.\frac{1}{2} g h\right|_{\eta_{o}} ^{2} & 0 \\
0 & \left.\frac{1}{2} g h\right|_{\eta_{o}} ^{2}
\end{array}\right]
$$

where $\mathbf{U}_{i}^{*}$ is the intermediate value of $\mathbf{U}_{i}$ (i.e. fractional step), $A_{i}$ is the area of cell $\Omega_{i}, \Delta t$ is the time step, the superscript $n$ represents the time level, subindex $k$ is used to denote the $k$-th edge of a cell, $l_{k}$ is the length of edge 
$k, \mathbf{E}^{*}=\left(\mathbf{F}^{*}, \mathbf{G}^{*}\right)$ represents the numerical approximation to $\mathbf{E}$, and $\left.h\right|_{\eta_{o}}$ is the depth considering a piecewise constant free-surface elevation (Valiani and Begnudelli, 2006). The numerical fluxes $\mathbf{F}^{*}$ and $\mathbf{G}^{*}$ are computed using the central-upwind method of Kurganov and Petrova (2004). In the second step the friction term is accounted to update the solution to time level $n+1$ from the values of $\mathbf{U}_{i}^{*}$. Friction slope components $S_{f x}$ and $S_{f y}$ are computed using Manning's equation

$$
S_{f x}=\frac{n^{2} u\|\mathbf{u}\|}{h^{4 / 3}} \quad S_{f y}=\frac{n^{2} v\|\mathbf{u}\|}{h^{4 / 3}}
$$

where $n$ is the Manning's coefficient and $\|\mathbf{u}\|$ is the $l^{2}$-norm of the velocity vector $\mathbf{u}$. It is widely recognised that at very shallow depths, an explicit discretisation of the friction terms can cause an overshooting of friction that often leads to source term instability. In order to avoid this problem, time integration of the friction term is performed using an implicit scheme widely adopted by other shallow-water models (e.g. Yoon and Kang, 2004; Sanders, 2008; Liang and Marche, 2009; de Almeida et al, 2012):

$$
\begin{aligned}
(h u)_{i}^{n+1} & =\frac{(h u)_{i}^{*}}{1+\Delta \operatorname{tg}\left[n^{2}\|\mathbf{u}\| /(h)^{4 / 3}\right]_{i}^{n}} \\
(h v)_{i}^{n+1} & =\frac{(h v)_{i}^{*}}{1+\Delta \operatorname{tg}\left[n^{2}\|\mathbf{u}\| /(h)^{4 / 3}\right]_{i}^{n}}
\end{aligned}
$$

Free-surface reconstruction and wetting and drying are handled by the volume/free-surface method (VFR) of Begnudelli and Sanders (2006), which provides a second-order accurate representation of the bed topography (Begnudelli and Sanders, 2006; Begnudelli et al., 2008). This further enhances the accuracy in the description of the terrain given by the extremely fineresolution topography used in this paper. The stability of the model is controlled by the standard Courant-Friedrichs-Lewy (CFL) condition.

The model includes only the surface component of urban drainage. This allows us to separate the influence of the urban terrain on the flood inundation from the rather complex interactions that can take place between surface and the sewerage flows. While a realistic representation of real world inundation requires the dynamic coupling of the two processes (Mark et al, 2004; Schmitt et al., 2004; Aronica and Lanza, 2005; Nasello and Tucciarelli, 2005; Maksimovic et al, 2009; Bazin et al, 2014), the study of the surface component alone is appropriate for the objectives of the present analysis. 


\section{Test cases}

A set of four different topographies have been used to analyse the influence of small scale changes in urban topography on the dynamics and final distribution of flooding. The tests use a $10 \mathrm{~cm}$ resolution digital elevation model produced from terrestrial LiDAR data collected by the Environment Agency of England and Wales (Ozdemir et al, 2013) in the urban area of Alcester (Warwickshire, UK), which is shown in Figure 2.a. The computational mesh generated using this DEM is composed of 3, 575, 123 nodes, 10, 711, 014 edges and 7, 135, 888 triangular elements. Figure 3 shows this computational mesh close to a street junction, illustrating how fine scale elements such as curbs are represented in the model. Such a fine resolution terrain model captures the shape of road cambers extremely accurately (as shown by Ozdemir et al, 2013), and the use of a second order model for the bed slope terms (in which the terrain is represented as inclined, rather than horizontal triangles, as described in Begnudelli and Sanders, 2006 and Begnudelli et al., 2008) brings the level of model representation of topography to a unprecedented level.

Small scale modifications have been introduced to the original topography in the two regions of the domain indicated with ellipses in Figure 2.a. These modifications have been strategically defined from previous observations of the simulations using the undisturbed topography. Namely, the combined inspection of the road topography, topology and the characteristics of the flood propagation indicated potential regions of the domain where the effect of topographical manipulations could lead to significant changes in the evolution and final distribution of flooded areas. The extent and magnitude of these alterations can be observed by comparing Figures 2.b and 2.d against Figures 2.c and 2.e, respectively. In the first of these modifications, the elevation of the road in Figure 2.b is reduced over a distance of approximately $30 \mathrm{~m}$ and by a maximum value of $18 \mathrm{~cm}$ (Figure 2.c). The second alteration was the introduction of a short hump (placed perpendicularly to the road direction and spanning from curb to curb) that increases the road elevation by a maximum value of $12 \mathrm{~cm}$ (from Figure 2.d to 2.e). Finally, a third scenario was generated by combining these two modifications into one DEM. Along with the original DEM, this provides four different scenarios that can be compared to analyse the influence of decimetric scale changes of the topography on inundation dynamics. These topographies will hereafter be referred to as A (unmodified topography), B (alteration shown in Figure 2.c), C (alteration 
shown in Figure 2.e) and D (the combination of terrain modifications shown in Figures 2.c and 2.e). All scenarios use exactly the same mesh topology, and only differ in the elevation of the road in the specific areas of the domain described above.

Two flow boundary conditions were used in the simulations. The first follows that previously adopted and described by Ozdemir et al (2013), which was derived by assuming a 200-year return period 30-min rainfall that is collected over a drainage area upstream of the inflow point. The discharge increases linearly from $0 \mathrm{~m}^{3} \mathrm{~s}^{-1}$ to the peak value $\left(0.35 \mathrm{~m}^{3} \mathrm{~s}^{-1}\right)$ during the first $7.5 \mathrm{~min}$, is kept constant for the subsequent $15 \mathrm{~min}$, after which it falls linearly to $0 \mathrm{~m}^{3} \mathrm{~s}^{-1}$ during the final $7.5 \mathrm{~min}$ (Figure 4). This boundary condition is uniformly distributed across the road situated on the North-East end of the computational domain in Figure 2.a. All other boundary edges were set as solid walls, except at roads and pavements, where they were set as open boundaries $(\partial \mathbf{U} / \partial \mathbf{n}=0)$. The second set of boundary conditions was obtained by multiplying the above hydrograph by 1.5 (peak discharge of $0.525 \mathrm{~m}^{3} \mathrm{~s}^{-1}$ ) while maintaining all other boundaries unchanged. The two different choices for the inflow boundary conditions will hereafter be referred to as $\mathrm{BC} 1$ and $\mathrm{BC} 2$ respectively. In all simulations the value of Manning's coefficient was set to $n=0.013$ for roads and pavements, and $n=0.035$ elsewhere. Two groups (i.e. $\mathrm{BC} 1$ and $\mathrm{BC} 2$ ) of four simulations each (i.e. using the four topographies previously described) were performed.

\section{Results}

Figure 5 shows the results of the group of simulations performed with $\mathrm{BC} 1$ at $t=12,30$ and $60 \mathrm{~min}$. Figures 5.a, 5.b, 5.c and 5.d, respectively represent simulations with topographies A, B, C and D. In all simulations the flood wave initially propagates southward along the main road located on the East side of the domain. As the water reaches street junctions, part of the flow can be diverted to side streets, depending on the local topography of the junction and neighbouring streets. For example, in Figure 5.a, the water passes by the first junction without being diverted. However, Figure $5 . \mathrm{b}$ shows that the reprofiling of the side street $(-18 \mathrm{~cm}$ as presented in Figure 2.c) allows the water to flow along North-West direction, inundating a region of the domain that is dry during the simulation performed with the original topography (Figure 5.a). A second flow diversion is also observed as the water reaches the central part of the domain, resulting in inundation at 
the topographical depression in the end of the street (center-west in Figures 5.a and b). This effect is considerably attenuated by the introduction of the $12 \mathrm{~cm}$ hump, as shown in Figure 5.c (e.g. at $t=30$ and $60 \mathrm{~min}$ ). The partial blockage of this street diversion by the hump also leads to more water being routed along the main road. This increased flow is now capable of overcoming the topographical blockage in the next downstream junction, allowing part of the flood wave to be diverted to the next street (as can be observed by comparing Figure 5.a against 5.c at 30 and $60 \mathrm{~min}$. The hump therefore mitigates flooding in one region of the domain at the expense of flooding areas that would otherwise be kept dry. A similar (although opposite) effect occurs as a result of the diversion of part of the flood water towards the NorthWest part of the domain shown in Figure 5.b, which results in a decrease in the volume of flow that is routed along the main road towards the South of the domain. However, in this example the flow reduction does not produce significant changes in the areas flooded downstream. The combined effects of these two modifications of the topography on the flooded areas is evidenced in Figure 5.d, which shows that only a negligible volume of the flood is diverted towards the central part of the domain compared to the corresponding results in Figure 5.a. In other words, two targeted minor alterations of the urban topography were able to completely prevent the inundation of a part of the domain that would otherwise receive a significant proportion of the flood flow. The results of these simulations also show that the fine scale model often captures the type of flow that occurs at low depths, when the water flows exclusively close to the curbs (e.g. gutters), and does not inundate the crown of the road camber.

Figure 6 shows the results of the simulations performed considering a higher flow scenario (BC2 boundary condition) for the four topographies and neglecting sinks. The propagation of the flood wave observed in this figure is similar to that presented in Figure 5 although flow depths and flooded areas are in general larger as a result of the increased flow rates. These results confirm the high influence of the topography alterations on the dynamics of flood inundation, as previously observed. Even though the combination of the two modifications (Figure 6.d) are not capable of completely preventing the inundation of the street located in the central region, it considerably reduces its effect. For example, it can be observed from Figures 6.c and 6.d that at $t=30 \mathrm{~min}$ the water overtopping the hump flows along the street and accumulates in the lowest region; however, this effect is considerably less pronounced in 6.d than in 6.a. The increase in the downstream hazards 
induced by the $12 \mathrm{~cm}$ hump can also be observed by comparing Figures 5.c and 6.c.

\section{Discussion}

The results of the 8 simulations presented in section 4 show that model predictions of surface water flood in urban areas are highly sensitive to decimetric-scale features of the urban topography. In particular, the road topography close to junctions dictate whether diversions will occur, and therefore plays a crucial role in the dynamics and final distribution of flooded areas. It has been observed that a minor (i.e. $18 \mathrm{~cm}$ ) and localized reduction of the road elevation can lead to significant inundation of areas that would otherwise not flood, while a small increase in the elevation (i.e. $12 \mathrm{~cm}$ ) can significantly reduce the impacts of flood inundation over large parts of the urban domain.

The sensitivity of flood inundation to decimetric scale topography poses significant challenges for accurate assessments of flood risk in urban areas. First, it confirms the importance of high-resolution topographical datasets on the quality of model predictions, as previously indicated by Ozdemir et al (2013). This puts particular pressure on computational resources and methods. Secondly, it also raises questions on the accuracy that is needed for the vertical position of topography datasets. Currently, terrain elevation data derived from airborne LiDAR that is usually used in flood risk assessment has a vertical accuracy of approximately 5 to $15 \mathrm{~cm}$. While our results show that systematic elevation errors of this magnitude can have a significant impact on predictions of flood risk, it is unclear how randomly distributed measurement errors may affect the results.

The complexity of the inundation processes observed in the simulations, combined with the sensitivity of the results to small changes, also reaffirms standing questions on the limitations of simplified approaches adopted to modeling urban flooding. For instance, at shallow depths water typically flows exclusively along gutters, which operate as two separate and independent channels. With increasing depths, the flow eventually overtops the crown of the road camber and the two separate channels merge into a single cross section. This behavior cannot be captured by 1D models, nor can it be reproduced by currently available sub-grid approaches.

While, on the one hand, the issues discussed above pose serious challenges for accurate modeling of floods in urban areas, they also unveil new 
opportunities for flood risk management. Namely, it has been shown that the final distribution of flood hazards can be significantly manipulated by introducing very small and localized changes to the topography of the road network. While it has been observed that alleviating harzards at particular areas can lead to increased inundation downstream (or vice-versa), an overall risk reduction can be obtained by selectively alleviating areas where the damage caused by flooding is highest. For example, the urban surface can be engineered to divert flood waters away from critical parts of the urban area towards zones where the expected damage is limited or non-existent (e.g. parks or green areas). The possibility of using the road network as efficient open-channels to transport excess flood waters across the domain could provide a new set of engineering techniques to expand current methods used in urban drainage (which are largely limited to the function of delivering water to the sewer system). Such approach would fill an existing gap in flood risk management, which lacks cost-effective measures to mitigate the impacts of medium to extreme storm events. While high-frequency, low magnitude events can usually be tackled by a combination of traditional (e.g. sewer system design) methods and SuDS (e.g. soakaways, green roofs, pervious surfaces, etc), these will often have only a minor effect on large flooding disasters, and expanding these systems to accommodate larger events is unlikely to be cost-effective. Our results show that only minor changes in the urban topography are needed to drive significant changes to the impacts, which suggests that low cost risk mitigation could be achieved under this proposed framework.

\section{Summary and conclusions}

This article analyzes the influence of small changes in the topography of the urban terrain on the propagation and final distribution of flooding in urban areas. Numerical simulations have been performed using a highly accurate finite volume shallow water model and an extremely fine resolution (i.e. $10 \mathrm{~cm}$ ) topography of a real urban area in the United Kingdom. This provided an unprecedented level of detail in the representation of the dynamics of flood inundation over the urban terrain. Four different topography scenarios were produced by introducing minor (decimetric scale) modifications to the original urban topography. A total of 8 numerical simulations were performed using two different inflow boundary conditions. 
The results of these numerical experiments have shown that small alterations in the urban topography can lead to contrastingly different patterns of flood inundation. Namely, the combination of two targeted and minor modifications - whereby the elevation of the road has been locally lowered by $18 \mathrm{~cm}$ and raised by $12 \mathrm{~cm}$ - has almost completely prevented flooding from impacting a large proportion of the modelled domain.

The sensitivity of flood inundation to small changes in the urban topography gives rise to a number of challenges. First, capturing the effect of small scale features requires finely resolved data that is rarely available for the great majority of model simulations that are currently performed for practical engineering studies. Second, not only the resolution of the datasets is important, but the accuracy of the vertical position also becomes a issue of high relevance. Airborne LiDAR datasets currently available have a vertical accuracy of approximately 5 to $15 \mathrm{~cm}$, which is of the same order of magnitude as typical depths that occur when overland flood flow is conveyed by road networks. Finally, the computational cost of modelling flood inundation at these scales is in general too high, or even unfeasible for most practical applications. This is particularly true when multiple simulations are required, which is typically the case in probabilistic risk assessments and engineering assessment of multiple scenarios.

While the dependency of flood inundation on small scale topography discussed above poses a number of practical difficulties to accurate assessments of flood risk, it also paves the way to new possibilities of risk mitigation that have not been explored to date. Namely, significant changes in the final distribution of flood hazards could be achieved by manipulating the topography at key regions of the urban domain. This could be used to divert part of the flood flow away from critical parts of the urban areas, or to guide the flood wave towards low impact zones (e.g. parks). As our results illustrate, only minor and localized modifications in the topography may be needed to produce substantial change to flood hazards, indicating that considerable mitigation can be achieved at low cost. The simulation results presented in this article also suggest that alterations in the road topography nearby road junctions can be particularly effective in producing major changes in the dynamics of flood propagation. This is because in these areas the local topography dictates how much water is diverted towards different parts of the urban domain, and therefore plays a crucial role in the aftermath of the urban flood.

The challenges and opportunities highlighted in this article are inher- 
ently interrelated. The level of detail needed for the design and optimization of the surface drainage methods proposed above can only be achieved in practice by enhanced availability of high-quality topographical data and high-performance computational resources and techniques.

Finally real-world urban flood inundation can be influenced by a number of issues that are not taken into account in our numerical analysis, including complex interactions with the sewer system. While the results presented in this article provide evidence of the influence of small scale topography on the surface component of inundation, further research is needed to understand potentially important interactions between these mechanisms and the sewerage system.

\section{Acknowledgements and data access information}

The Environment Agency of England and Wales (EA) is acknowledged for providing the terrestrial LiDAR data used in this article. This data is copyrighted and can be requested under licence from the EA (www.environmentagency.gov.uk). All model results will be made available under request to the corresponding author.

\section{References}

Aronica, G. T. and L. G. Lanza (2005), Drainage efficiency in urban areas: a case study, Hydrological Processes, 19 1105?1119, DOI: 10.1002/hyp.5648

Bates, P. D., M. S. Horritt, and T. J. Fewtrell (2010), A simple inertial for- mulation of the shallow water equations for efficient two-dimensional flood inundation modelling,, J. Hydrol, 387 33-45, doi:10.1016/ j.jhydrol.2010.03.027.

Bazin, P-H, Nakagawa, H., Kawaike, K., Paquier, A. and E. Mignot (2014), Modeling Flow Exchanges between a Strees and an Underground Drainage Pipe during Urban Floods, Journal of Hydraulic Engineering, 140 No. 10, 04014051

Begnudelli, L. and B. Sanders (2006), Unstructured Grid Finite-Volume Algorithm for Shallow-Water Flow and Scalar Transport with Wetting and Drying, Journal of Hydraulic Engineering, 132 No. 4, 371-384 
Begnudelli, L., Sanders, B. F., and S. F. Bradford (2008), Adaptive GodunovBased Model for Flood Simulation, Journal of Hydraulic Engineering, 134 No. 6, 714-725

Cunge J.A., Holly F.M. and A.Verwey (1980), Practical Aspects of Computational River Hydraulics. Pitman, London, U.K.

de Almeida, G. A. M., Bates, P. D., Freer, J., Souvignet, M. (2012), Improving the stability of a simple formulation of the shallow water equations for 2D flood modelling. Water Resources Research, VOL. 48, doi:10.1029/2011WR011570

de Almeida, G. A. M. and P. D. Bates (2013), Applicability of the local inertial approximation of the shallow water equations to flood modeling Water Resources Research, VOL. 49 , 1?12, doi:10.1002/wrcr.20366

DEFRA, 2008. Future Water: The Government?s Water Strategy for England. CM7319 London.

Delleur, J. W. (2003), The Evolution of Urban Hydrology Journal of Hydraulic Engineering, 129, 563-573

Djordjevic S. Prodanovic D. and Maksimovic (1999), An approach to simulation of dual drainage. Water Science and Technology, 39(9) 95-103

Dottori, F., Di Baldassarre, G. and E. Todini (2013), Detailed data is welcome, but with a pinch of salt: Accuracy, precision, and uncertainty in flood modeling. Water Resources Research, 49 6079-6085

Environment Agency, 2009. Flooding in England: A National Assessment of Flood Risk, Environment Agency, Bristol, UK.

Evans, E. P., Simm, J. D., thorne, C. R., Arnell, N. W., Ashley, R. M., Hess, T. M., Lane, S. N., Morries, J., Nicholls, R. J., Penning-Rowsell, E. C., Reynard, N. S., Saul, A. J., Tapsell, S. M., Watkinson A. R. and H. S. Whether (2008), An update of the Foresight Future Flooding 2004 qualitative risk analysis. Cabinet Office, London.

Fang, Xing and D. Su (2005), An integrated one-dimensional and twodimensional urban stormwater flood simulation model Journal of the American Water Resources Association, 42(3), 713-724. 
Gallegos, H. A., Schibert, J. E. and B. F. Sanders (2009), Two-dimensional, high-resolution modeling of urban dam-break flooding: A case study of Balwin Hills, California Advances in Water Resources, 32, 1323-1335.

Gallien, T. W., Schibert, J. E. and B. F. Sanders (2011), Predicting tidal flooding of urbanised embayments: A modeling framework and data requirements. Coastal Engineering, 58, 567-577.

Guinot, V. (2012), Multiple porosity shallow water models for macroscopic modelling of urban floods. Advances in Water Resources, 37, 40-72.

Kurganov, A. and G. Petrova (2004), Central-Upwind Schemes on Triangular Grids for Hyperbolic Systems of conservation Laws. Numerical Methods for Partial Differential Equations, 21, pp. 536-552

Leandro, J. Chen, A., Djordjevic, S. and D. A. Savic (2009), Comparison of 1D/1D and 1D/2D Coupled (Sewer/Surface) Hydraulic Models for Urban Flood Simulation. Journal of Hydraulic Engineering, 135, No. 6 pp. 495504

LeVeque, R. J. (2002), Finite Volume Methods for Hyperbolic Problems, 257 pp., Cambridge Univ. Press, Cambridge, Mass.

Liang, Q. and F. Marche (2009), Numerical resolution of well-balanced shallow water equations with complex source terms Advances in Water Resources, 32, 873-884

Maksimovic, C., Prodanovic, D., Boonya-Aroonnet, S., Leitao, J. P., Djordjevic, S., and R. Allitt (2009), Overland flow and pathway analysis for modelling of urban pluvial flooding Journal of Hydraulic Research, 47, $512-523$

Mark, O., Weesakul, S., Apirumanekul, C., Boonya-Aroonnet, S. and S. Djordjevic (2004), Potential and limitations of 1D modelling of urban flooding Journal of Hydrology, 299, 284-299

Mignot, E., Paquier, A. and S. Haider (2006), Modeling floods in a dense urban area using 2D shallow water equations. Journal of Hydrology, 327, $186-199$ 
Molinaro, P., Di Filippo, A., and F. Ferrari (1994), Modelling of flood wave propagation over flat dry areas of complex topography in presence of different infrastructures. In Proceedings of Specialty Conference on "Modelling of flood propagation over initially dry areas", Milan, 20-30 June; 209-225

Nasello, C. and T. Tucciarelli (2005), Dual Multilevel Urban Drainage Model Journal of Hydraulic Engineering, 131, No. 9, 748-754

Ozdemir, H., Sampson, C. C., de Almeida, G. A. M., and P. D. Bates (2013), Evaluating scale and roughness effects in urban flood modelling using terrestrial LiDAR data Hydrol. Earth Syst. Sci., 17, 4015-4030

Sampson, C., Fewtrell, T. J., Duncan, A., Shaad, K., Horritt, M. S. and P. D. Bates (2012), Use of terrestrial laser scanning data to drive decimetric resolution urban inundation models, Advances in Water Resources, Vol. $41,1-17$

Sanders, B. (2008), Integration of a shallow water model with a local time step, Journal of Hydraulic Research, Vol. 46, No. 4, 466-475, doi:10.3826/jhr.2008.3243

Sanders, B., Schubert, J. E. and H. A. Gallegos (2008), Integral formulation of shallow-water equations with anisotropic porosity for urban flood modeling, Journal of Hydrology, 362, 19-38

Soares-Frazao, S, Lhomme, J., Guinot, V., and Y. Zech (2008), Twodimensional shallow-water model with porosity for urban flood modelling, Journal of Hydraulic Research, Vol. 46, No. 1, 45-64

Schmitt, T. G., Thomas, M. and N. Ettrich (2004), Analysis and modelling of flooding in urban drainage systems, Journal of Hydrology, 299, 300-311

Valiani, A. and L. Begnudelli (2006), Divergence form for bed slope source term in shallow water equations, Journal of Hydraulic Engineering, 132 No. 7 , pp. $652-665$

Yoon, T. H. and S-K. Kang. (2004), Finite Volume for Two-Dimensional Shallow Water Flows on Unstructured Grids, Journal of Hydraulic Engineering, 130 No. 7, 678-688 


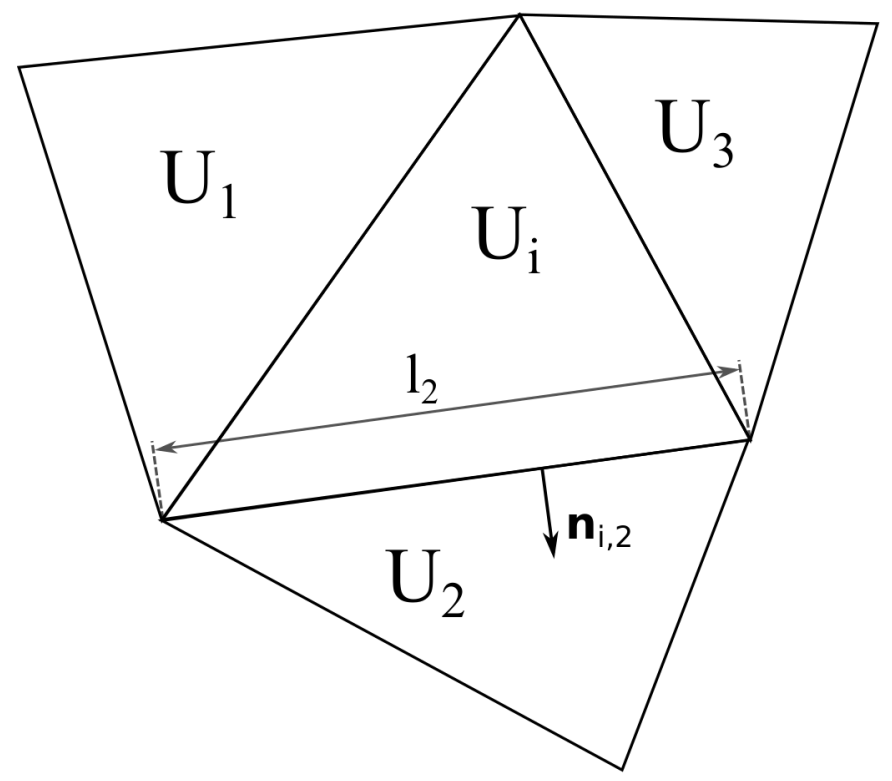

Figure 1: Ustructured computational mesh variables. 


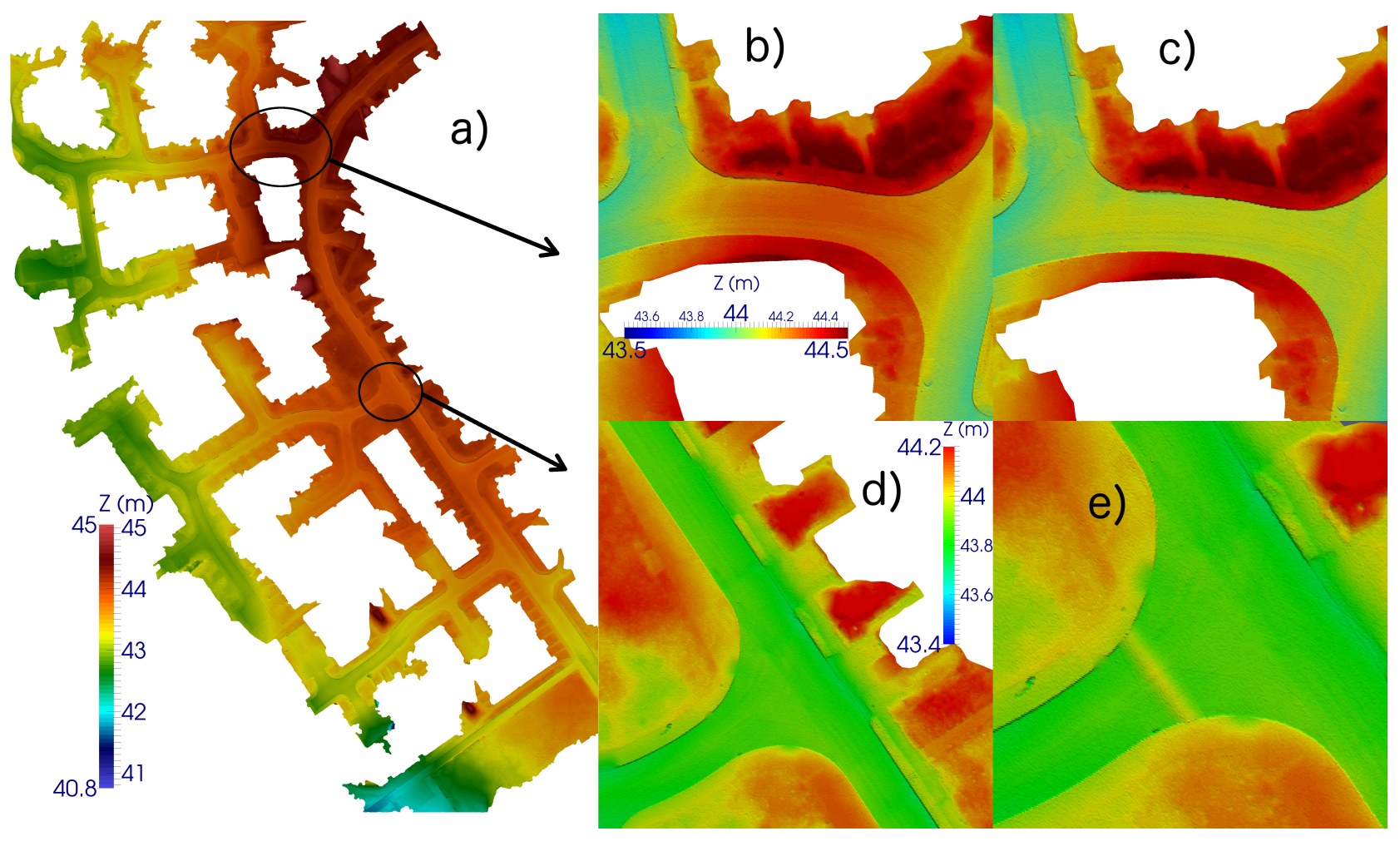

Figure 2: Original and modified DEMs. a) original DEM; $b$ and d) zoom of the two regions indicated in the original DEM; c and e) modified DEMs.

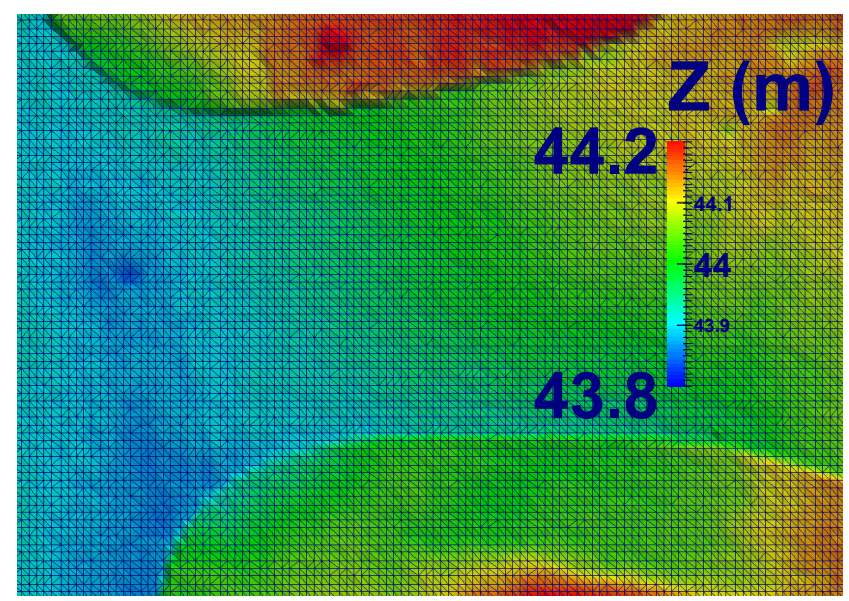

Figure 3: Detail of the computational mesh used. 


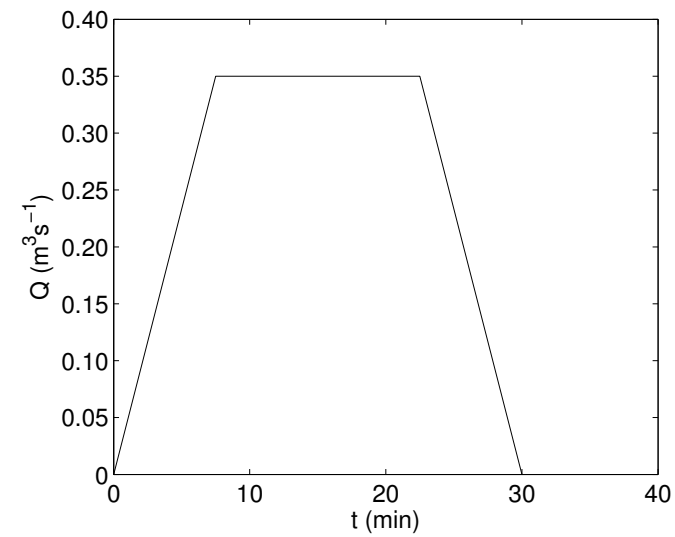

Figure 4: Hydrograph used as the upstream boundary condition in BC1. 

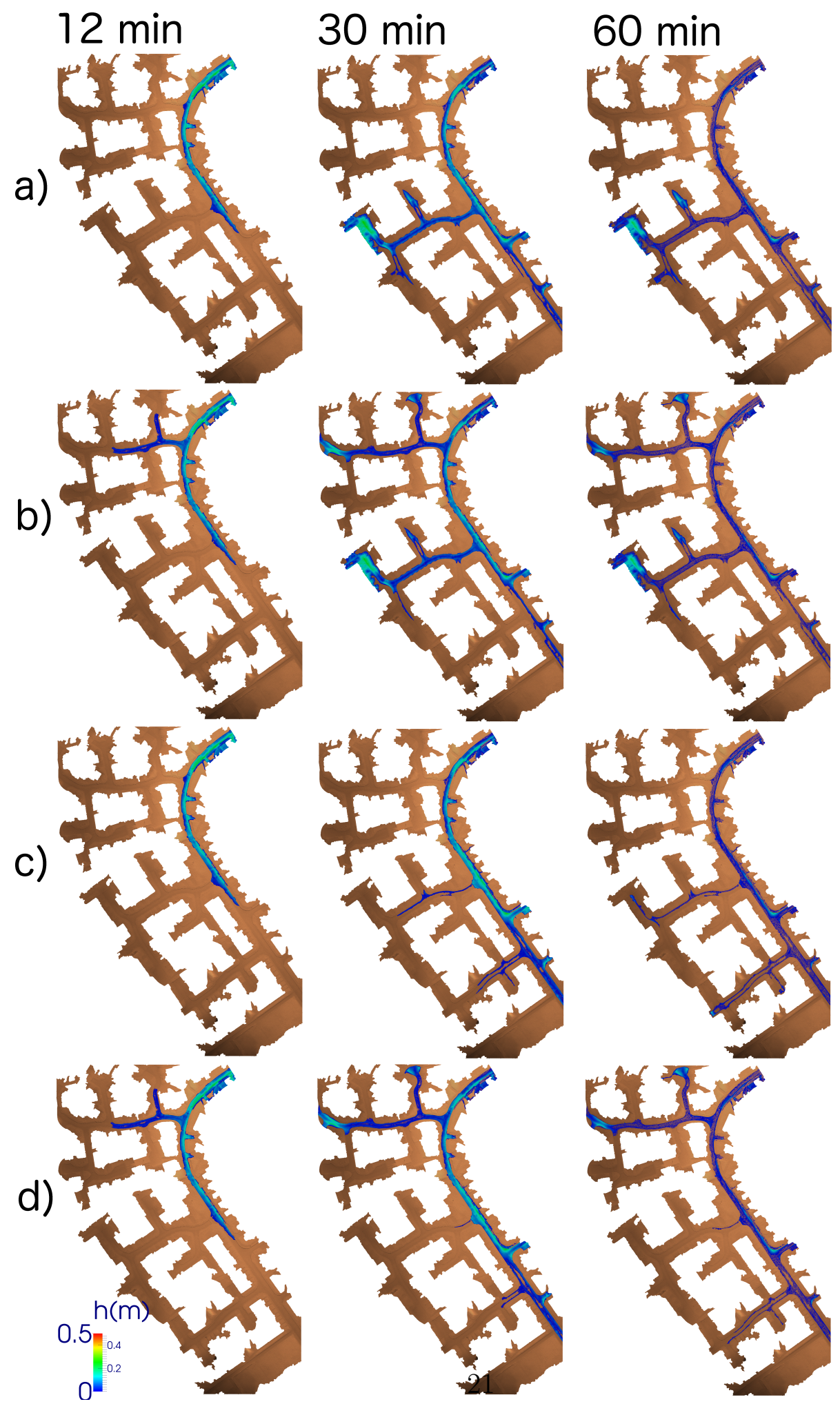

Figure 5: Results of the simulations using BC1 boundary conditions and neglecting the sewerage system. Results are shown at $t=12,30$ and 60 min and for the four scenarios. a) original topography; b) DEM modification corresponding Figure 1.c; c) DEM modification shown in Figure 1.e; d) combination of the two modifications. 
a)
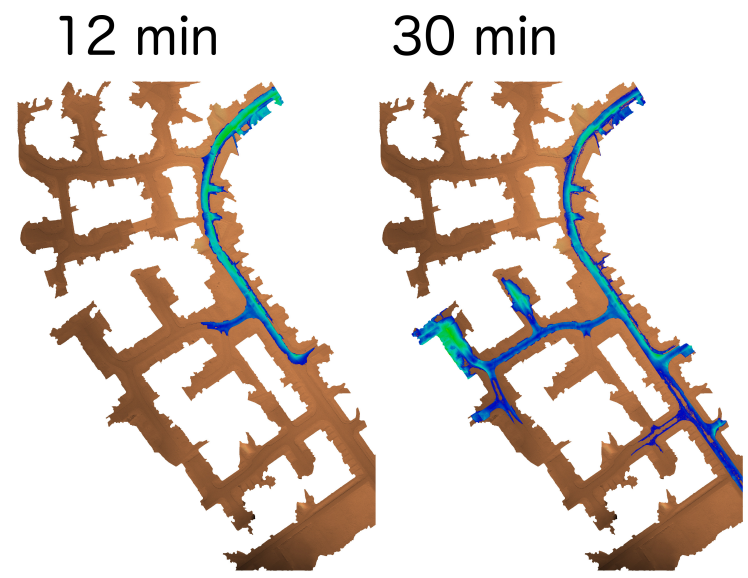

$60 \min$

b)
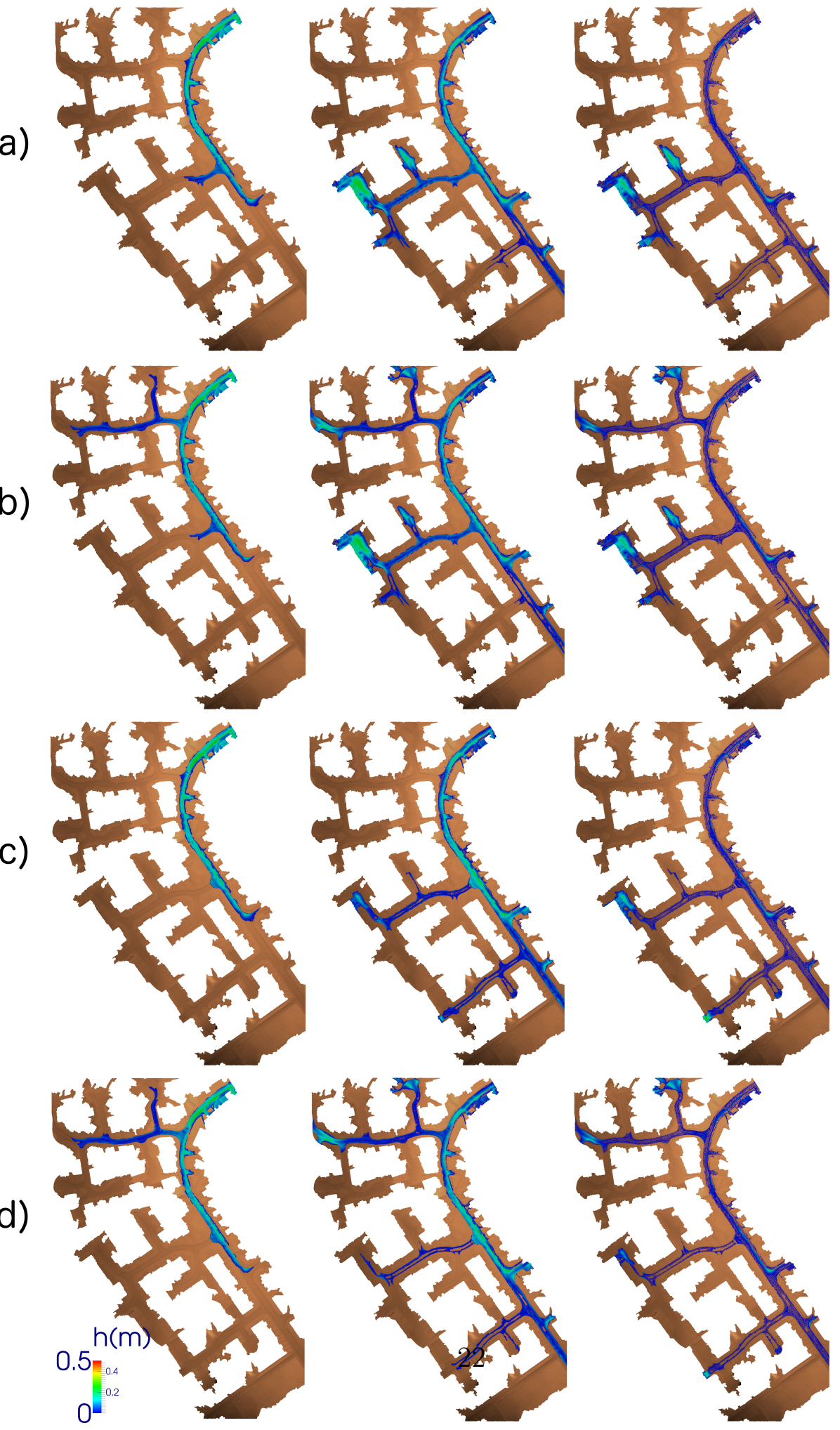

Figure 6: Results of the simulations using $\mathrm{BC} 2$ boundary conditions and neglecting the sewerage system. Results are shown at $t=12,30$ and 60 min and for the four scenarios. a) original topography; b) DEM modification corresponding Figure 1.c; c) DEM modification shown in Figure 1.e; d) combination of the two modifications. 